\title{
Standard Schnauzer
}

National Cancer Institute

\section{Source}

National Cancer Institute. Standard Schnauzer. NCI Thesaurus. Code C53772.

The Standard Schnauzer is a medium-sized, rugged, robust dog with bushy eyebrows, whiskers and a beard. The feet are small and cat-like, with arched toes. It has a harsh, wiry outer coat and dense, soft undercoat. The coat comes in salt and pepper or solid black. Height: 17-20 inches (43-51 cm.) Weight: 30-45 pounds (14-20 kg.) 